\title{
Rectal Gastrointestinal Stromal Tumor (GIST): About a Rare and Unusual Case
}

\author{
Abderrahmane Jallouli ${ }^{*}$, Mariama Jarti ${ }^{1}$, Marj Zohour Haida ${ }^{1}$, Mouna El Bouatmani ${ }^{1}$, Adil Ait Errami ${ }^{1}$, \\ Sofia Oubaha ${ }^{2}$, Zouhour Samlani ${ }^{1}$, Khadija Krati ${ }^{1}$ \\ ${ }^{1}$ Hepato-Gastroenterology Department, University Hospital of Mohammed VI, Marrakesh, Morocco \\ ${ }^{2}$ Physiology Laboratory, Faculty of Medicine and Pharmacy, Cadi Ayyad University, Marrakech, Morocco \\ * Corresponding author: Abderrahmane Jallouli; abderrahmanejallouli@ gmail.com
}

Received 10 September 2021;

Accepted 05 November 2021;

Published 14 November 2021

\begin{abstract}
Rectal gastrointestinal stromal tumors (GIST) are extremely rare, accounting for approximately $0.1 \%$ of all rectal tumors. Diagnosis is based on histological and immunohistochemical confirmation.

We report the case of a 38-year-old patient with a rectal GIST revealed by chronic rectal bleeding associated with rectal tenesmus, abdominal pain with painful contractions and frequent evacuations evolving in a context of altered general state. Rectoscopy was used to visualize the tumor mass and to take biopsies; their histological and immunohistochemical study revealed a rectal location of a gastrointestinal stromal tumor. The extension assessment was negative. After discussion of the case in a multidisciplinary consultation meeting, management consisted of initially putting the patient on Imatinib given the large tumor size, the degree of local invasion and the location of the mass (lower rectum), with regular follow-up in order to schedule a less invasive surgical resection later.

Despite the rarity of rectal GIST, early diagnosis is necessary to avoid progression to locoregional invasion complicating some surgical resections given the anatomical constraints of the pelvic region. Hence the interest of neoadjuvant therapy with tyrosine kinase inhibitors allowing in some cases a decrease in tumor volume, a regression of the degree of local invasion and a decrease in the morbidity of the surgery in order to improve the patient's quality of life.
\end{abstract}

Keywords: gastrointestinal stromal tumors, GIST, rectal tumor, Imatinib, rectal bleeding

\section{Introduction}

Gastrointestinal stromal tumors (GIST) constitute $1 \%$ of all gastrointestinal tumors ${ }^{[1]}$, and are found mainly in the stomach and intestine. Rectal localization, on the other hand, is very rare, accounting for $0.1 \%$ of all rectal tumors ${ }^{[2,3]}$. GIST usually expresses the CD-117 receptor (Kit), and less frequently the CD-34 receptor ${ }^{[3-5]}$. The interest of evoking this entity is to improve the prognosis of patients with this rare form of rectal tumor.

We report a particular observation of rectal GIST in a $38-$ year-old patient, revealed by chronic rectal bleeding.

\section{Observation}

\section{Patient Information}

A 38-year-old patient, with a history of chronic active smoking for 20 years and occasional alcoholism for 2 years, has complained for a year of rectal bleeding associated with frequent mucous evacuations, rectal tenesmus and abdominal pain with painful contractions. Evolving in a context of marked alteration of the general state (weight loss of $6 \mathrm{~kg}$, asthenia and anorexia).

\section{Clinical Findings}

Clinical examination on admission showed mucocutaneous pallor. The abdominal examination was normal. The digital rectal examination found a fixed posterior hemi-circumferential mass 2.5 $\mathrm{cm}$ from the anal margin, the glove came back stained with blood. The rest of the somatic examination, notably the examination of the lymph nodes and the examination of the respiratory system, was without anomalies.

\section{Diagnostic Assessment}

A rectoscopy had revealed a process at $2.5 \mathrm{~cm}$ from the anal margin that bled easily on contact with the deep plane, measuring about $6 \mathrm{~cm}$ (Figure 1). 


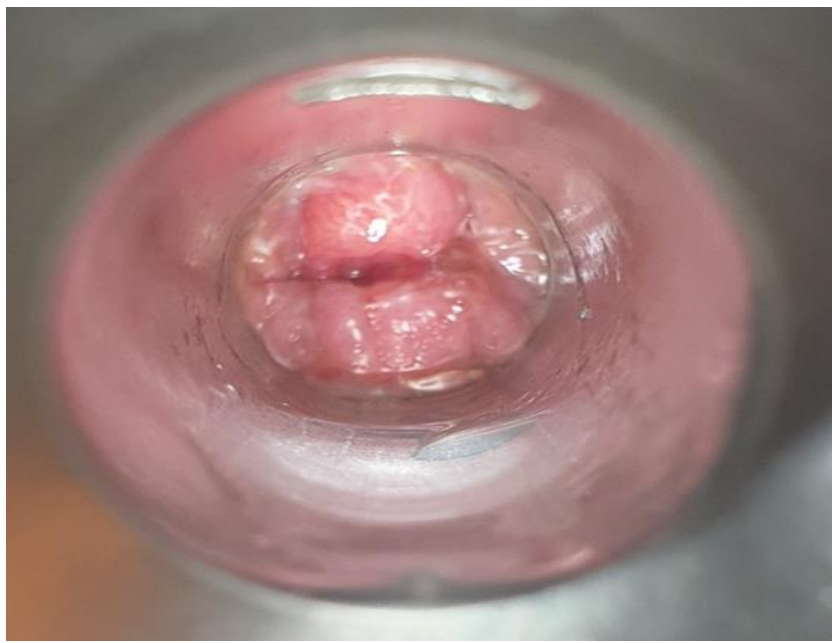

Figure 1: Rectoscopy: a posterior mass at $2.5 \mathrm{~cm}$ from the anal margin

The biopsy of this process showed a mesenchymal proliferation made of long intertwined bundles, with spindle-shaped tumor cells with elongated nuclei with fine nucleated chromatin in some places, the cytoplasm is eosinophilic, the stroma is fibroinflammatory and rich in eosinophilic polynuclei, and a hemangiopericytic vascularization. A complementary immunohistochemical study showed a diffuse and intense cytoplasmic expression of tumor cells of the anti-CD 117 antibody, a moderate nuclear expression of $30 \%$ of tumor cells of the anti-Ki 67 antibody with absence of expression of tumor cells of the anti AML antibody, making an aspect compatible with a gastrointestinal stromal tumor (GIST). The extension assessment included a total colonoscopy which revealed a friable ulcerating tumor process bleeding easily on contact in the lower rectum, the rest of the colonic mucosa was without macroscopic abnormalities. Pelvic magnetic resonance imaging (MRI) showed a posterior parietal hemicircumferential lesional process of the lower and middle rectum stenosing in places measuring $6.7 \times 7.6 \times 7.3 \mathrm{~cm}$ with fairly well limited contours at $2.4 \mathrm{~cm}$ from the anal margin, with extra-mural and meso rectal extension with infiltration of meso recti fascia (circumferential resection margin at 0 ), coming in intimate contact with the ileo-coccygeal muscles and the right internal iliac vessels with respect to the fatty separation line, and infiltrating the internal sphincter over $8 \mathrm{~mm}$ in length and intimate contact with the external sphincter, without signs of extra-mural venous invasion (Figure 2).

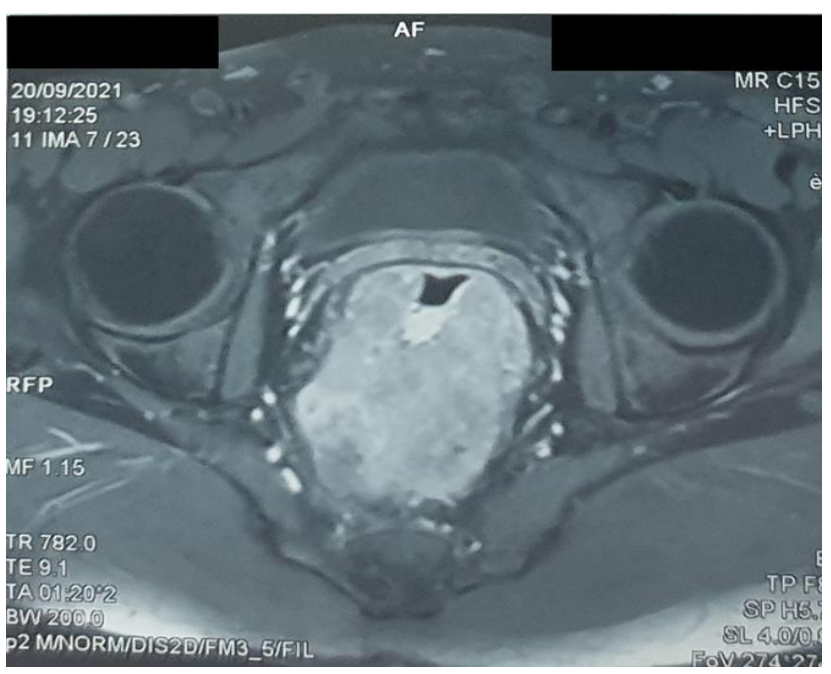

Figure 2: Pelvic MRI of the tumor
A thoraco-abdomino-pelvic computed tomography (CT) scan was performed and did not reveal any secondary localization.

\section{Therapeutic Intervention}

After discussion of the patient's file in a multidisciplinary consultation meeting (RCP), neoadjuvant treatment with Imatinib was indicated because of the mutilating nature of the surgical treatment (abdominoperineal resection (ARP)). However, less invasive secondary surgery can be considered if there is a good response to Imatinib by reducing the tumor volume.

\section{Discussion}

Gastrointestinal stromal tumors (GIST) are uncommon digestive mesenchymal tumors that develop from Cajal-like interstitial cells expressing the CD-117 proto-oncogene in $80 \%$ of cases ${ }^{[3-5]}$. They are preferentially located in the gastric (in about $70 \%$ of cases) and intestinal (in about $30 \%$ of cases) regions ${ }^{[2]}$. Rectal localization is exceptional; with an incidence of approximately $0.1 \%$ of all rectal tumors and accounting for approximately $5 \%$ of all GIST ${ }^{[2,3]}$. Rectal GIST occur in the majority of cases sporadically, although predisposing and familial forms have been described ${ }^{[6]}$.

The clinical signs do not differ from other histological types of rectal tumors. Rectal GIST may present with gastrointestinal hemorrhage (rectal bleeding), abnormal discharge (mucus, pus, etc.), transit disorders (including constipation), or altered general condition. These tumors can be diagnosed incidentally in asymptomatic patients (during a digital rectal examination, rectoscopy or discovered on radiological examination) ${ }^{[1,7,8]}$.

Rectoscopy plays an important role in the identification of rectal GIST, particularly in the endophytic forms, which are manifested by a rounded swelling that pushes back a normal or ulcerated mucosa. In contrast to exophytic forms of rectal GIST, which may appear on rectoscopy as a stiffening of the rectal wall [9].

Echo-endoscopy allows the detection of rectal GIST, which is most often a round or oval formation, which is located in the muscularis or submucosa. It allows a prognostic orientation of the tumor mass; by evaluating its size, its contours, a loco-regional infiltration. The presence of a tumor mass greater than $10 \mathrm{~cm}$, locoregional invasion, intra-tumoral cystic areas or central necrosis are signs suggestive of malignancy ${ }^{[10]}$. Echo-endoscopy also allows biopsies to be taken.

Immunohistochemistry of rectal GIST biopsy fragments obtained during endoscopy or echo-endoscopy is of major interest in making a positive diagnosis: they characteristically express CD117 or C-KIT (KIT receptor tyrosine kinase marker) receptors, and often CD-34 receptors. The positivity of these two receptors varies depending on the location of the tumor. GIST located along the gastrointestinal tract express CD-117, which also plays a therapeutic role; it is the target of Imatinib, which is a receptor tyrosine kinase inhibitor ${ }^{[3]}$.

CT with contrast injection is often the initial imaging technique of choice, both for detection of the tumor mass (GIST typically appear as large, well-circumscribed eccentric masses that enhance with intravenous contrast.) and for staging by looking for organ or lymphatic invasion, or liver metastases ${ }^{[1]}$.

On MRI, the tumor is usually hyposignal in T1 with welllimited borders and iso-signal in $\mathrm{T} 2{ }^{[7]}$, it is likewise useful, especially in patients with contraindications to CT, to study locoregional and distant (hepatic) invasion ${ }^{[11]}$. 
Given their potential for malignancy, surgical resection remains the only curative treatment for localized forms of rectal GIST ${ }^{[1]}$. The major goal of all surgical resections (transabdominal, trans-anal and endoscopic) is to obtain margins of healthy tissue without tumor invasion ${ }^{[12]}$. It is essential to avoid intraoperative perforation, which leads to peritoneal dissemination and a survival similar to that of patients with incomplete resection [13]. The choice of surgical approach must be made in multidisciplinary consultation and must be adapted to each patient and to the characteristics of each tumor (size, invasion, anal sphincter status, etc.) ${ }^{[14]}$. Lymph node dissection is not systematically performed because lymph node involvement is rare [8].

In addition to its unfavorable prognosis and the high risk of local recurrence equivalent to other GIST locations, the rectal location is characterized by its anatomical constraints (location in the pelvis), complicating certain surgical resections ${ }^{[1]}$. This is why Imatinib, which is a tyrosine kinase inhibitor, is of interest in the treatment protocol for rectal GIST. It should be considered in all cases requiring a reduction in tumor volume in order to reduce the morbidity of the surgical procedure and improve the patient's quality of life ${ }^{[15-17]}$. For metastatic forms, the treatment of choice remains Imatinib ${ }^{[12]}$.

In randomized trials, a $70 \%$ response rate for Imatinib has been noted in unresectable and metastatic GIST. Another study in 161 patients with locally advanced GIST showed a good response to neoadjuvant Imatinib therapy with an $80 \%$ response rate ${ }^{[18]}$.

Survival for patients with rectal GIST at 5 years is between $22 \%$ and $66 \%$ for high-grade and low-grade tumors, respectively. Tumors with a mitotic index greater than 5 per 10 fields and size greater than $10 \mathrm{~cm}$ are at high risk for recurrence ${ }^{[9]}$.

\section{Conclusion}

Rectal GIST are very rare. Diagnostic confirmation is based on anatomopathological and immunohistochemical studies. The standard treatment for localized forms is surgical resection. In cases of large tumor volume, obvious local invasion, and major morbidity associated with surgical treatment, neoadjuvant treatment with Imatinib may be indicated, followed by less invasive resection if there is a good response.

\section{Authors' Contributions}

All authors participated in the conception, drafting the work, critically revised the manuscript, approved the final version to be published, and agree to be accountable for all aspects of the work.

\section{Funding}

The author(s) received no financial support for the research, authorship, and/or publication of this article.

\section{Declaration of conflicting interests}

The author(s) declared no potential conflicts of interest with respect to the research, authorship, and/or publication of this article.

\section{References}

[1] Kane WJ, Friel CM. Diagnosis and Treatment of Rectal Gastrointestinal Stromal Tumors. Diseases of the Colon \& Rectum. 2019;62(5):537-40.

[2] Miettinen M, Majidi M, Lasota J. Pathology and diagnostic criteria of gastrointestinal stromal tumors (GISTs): a review. European Journal of Cancer. 2002;38:S39-51.

[3] Balu J, Subramanian S, P S, et al. A rectal gastrointestinal stromal tumor-a plea for neoadjuvant imatinib and TAMIS. J Coloproctol (Rio J). 2020;40(1):89-93.

[4] Downs-Kelly E, Rubin BP. Gastrointestinal Stromal Tumors: Molecular Mechanisms and Targeted Therapies. Pathology Research International. 2011;2011. doi:10.4061/2011/708596

[5] Joensuu H, Fletcher C, Dimitrijevic S, et al. Management of malignant gastrointestinal stromal tumours. The Lancet Oncology. 2002;3(11):655-64.

[6] Yantiss RK, Rosenberg AE, Sarran L, et al. Multiple gastrointestinal stromal tumors in type I neurofibromatosis: a pathologic and molecular study. Mod Pathol. 2005;18(4):475-84.

[7] Panzironi G, Manganaro L, Ricci F, et al. A case of rectal GIST: findings of MR-spiral CT imaging and transrectal ultrasound guided biopsy. European Journal of Radiology Extra. 2003;47(2):66-9.

[8] Elkihal L, Ajana F-Z, Seddik H, et al. Nouvelles acquisitions diagnostiques et thérapeutiques sur les tumeurs stromales digestives: à propos de cinq cas. Cahiers d'études et de recherches francophones / Santé. 2005;15(4):271-83.

[9] Samlani-Sebbane Z, Diffaa A, Charaf K, et al. Le GIST rectal: à propos de deux observations et une revue de la littérature. J Afr Hepato Gastroenterol. 2011;5(1):60-2.

[10] van den Berg JC, van Heesewijk JP, van Es HW. Malignant stromal tumour of the rectum: findings at endorectal ultrasound and MRI. BJR. 2000;73(873):1010-2.

[11] Kalkmann J, Zeile M, Antoch G, et al. Consensus report on the radiological management of patients with gastrointestinal stromal tumours (GIST): recommendations of the German GIST Imaging Working Group. Cancer Imaging. 2012;12(1):126-35.

[12] Kameyama H, Kanda T, Tajima Y, et al. Management of rectal gastrointestinal stromal tumor. Translational Gastroenterology and Hepatology. 2018;3. doi:10.21037/tgh.2018.01.08

[13] Demetri GD, von Mehren M, Antonescu CR, et al. NCCN Task Force Report: Update on the Management of Patients with Gastrointestinal Stromal Tumors. J Natl Compr Canc Netw. 2010;8(Suppl 2):S-1-S-41.

[14] Rejab H, Kridis WB, Ameur HB, et al. Tumeur stromale rectale: à propos d'une observation. Pan Afr Med J. 2014;17. doi:10.11604/pamj.2014.17.119.2285

[15] Hamada M, Ozaki K, Horimi T, et al. Recurrent rectal GIST resected successfully after preoperative chemotherapy with imatinib mesylate. Int J Clin Oncol. 2008;13(4):355-60.

[16] Fiore M, Palassini E, Fumagalli E, et al. Preoperative imatinib mesylate for unresectable or locally advanced primary gastrointestinal stromal tumors (GIST). European Journal of Surgical Oncology (EJSO). 2009;35(7):739-45. 
[17] Machlenkin S, Pinsk I, Tulchinsky H, et al. The effect of neoadjuvant Imatinib therapy on outcome and survival after rectal gastrointestinal stromal tumour: Neoadjuvant Imatinib therapy in rectal GIST. Colorectal Disease. 2011;13(10):1110-5.

[18] Lo SS, Papachristou GI, Finkelstein SD, et al. Neoadjuvant Imatinib in Gastrointestinal Stromal Tumor of the Rectum: Report of a Case. Diseases of the Colon \& Rectum. 2005;48(6):1316-9.
Open Access This article is licensed under a Creative Commons Attribution 4.0 International License, which permits use, sharing, adaptation, distribution and reproduction in any medium or format, as long as you give appropriate credit to the original author(s) and the source, provide a link to the Creative Commons license, and indicate if changes were made. The images or other third party material in this article are included in the article's Creative Commons license, unless indicated otherwise in a credit line to the material. If material is not included in the article's Creative Commons license and your intended use is not permitted by statutory regulation or exceeds the permitted use, you will need to obtain permission directly from the copyright holder. To view a copy of this license, visit https://creativecommons.org/licenses/by/4.0/.

(C) The Author(s) 2021 